\title{
SPECTROPHOTOMETRY OF M AND C STARS
}

\author{
K. NANDY \\ Royal Observatory, Edinburgh, Scotland \\ and \\ P. SMRIGLIO \\ Rome Observatory, Rome, Italy
}

\begin{abstract}
Summary. Surveys of $M$ and $C$ stars down to infrared magnitudes $12 \mathrm{~m} \cdot 0$ in two galactic regions in the direction Cygnus and anticentre are described. Infrared magnitudes are derived by a new method of calibration based on the use of a combination of crossed calcite plates and Polaroid HN32. Spectral classification is based on spectrophotometric criteria which measure the relative strengths of the molecular bands visible in the infrared in the low dispersion objective prism spectra. Infrared absolute magnitudes and interstellar extinction in the infrared are obtained from the present material and published data, and the space densities of $M$ stars are determined.

Full accounts have been published in Publ. Edinburgh Roy. Obs. 7, 1 (1970) and 7, 73 (1971).
\end{abstract}

